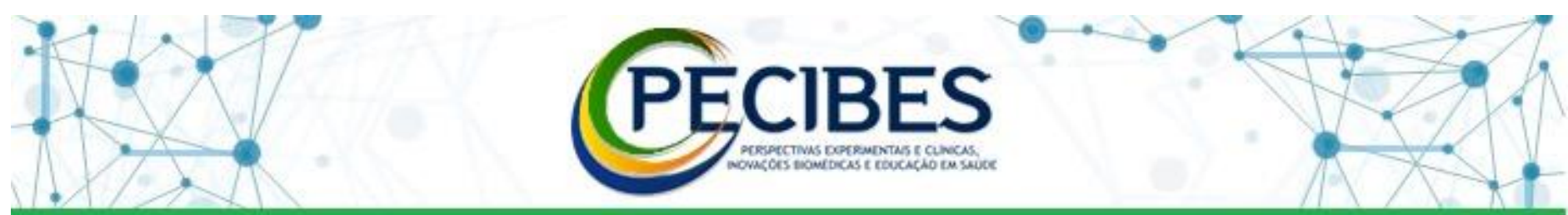

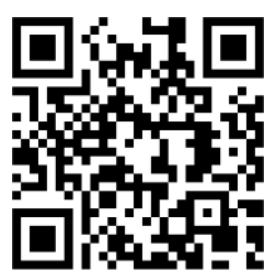

http://www.seer.ufms.br/ind ex.php/pecibes/index

*Autor

correspondente:

Erinéia Januário

Godoy, Federal de

Mato Grosso do Sul -

UFMS.

E-mail do autor: erineiagodoy@gmail.co $\mathrm{m}$

Descritores: Cuidados de Enfermagem.

Diabetes Mellitus. Hipertensão.

Alojamento Conjunto.

Integralidade em

Saúde.

Key-words: Nursing

Care.

DiabetesMellitus.

Hypertension.Roomin g-inCare.Integrality in Health.

Descriptores:

Enfermería. Diabetes

Mellitus.Hipertensión.

Alojamiento

Conjunto. Integralidad

en Salud.

\section{Sistematização da assistência de enfermagem à puérpera com diagnóstico de Diabetes Mellitus Tipo 1 e Hipertensão Arterial}

Systematization of Nursing Care for puerperal womem with Type 1 Diabetes Mellitus and Arterial Hypertension

Erinéia Januário Godoy ${ }^{1}$, Daniela Miyuki Sato ${ }^{1}$, Ana Paula de Assis Sales ${ }^{3}$.

1. Graduanda em Enfermagem. Universidade Federal de Mato Grosso do Sul. Campo Grande, MatoGrosso do Sul, Brasil.

2. Enfermeira. Departamento de Enfermagem da Universidade Federal de Mato Grosso do Sul. Campo Grande, Mato Grosso do Sul, Brasil

Introdução: no Diabetes Mellitus Tipo 1 há um déficit absoluto na produção do hormônio insulina. Esta patologia interfere no metabolismo dos carboidratos no processo gestacional, o que requer controle glicêmico. Enquanto a hipertensão ao lado da hemorragia grave e das infecções puerperais está associada às principais causas de morte materna no Brasil e no mundo, bem como risco importante à vida fetal. Logo, a Sistematização da Assistência de Enfermagem (SAE) possibilita organizar o trabalho do profissional enfermeiro, de modo que o mesmo realize um acompanhamento adequado das mulheres com estes agravos a fim de impedir complicações no puerpério. Objetivo: Descrever a SAE realizada a uma puérpera em hospital de ensino. Material e métodos: relato de caso baseado nos dados referentes ao registro da assistência de enfermagem. Para isso, verificaram-se os dados referentes às etapas do processo de enfermagem: coleta de dados, diagnóstico, planejamento, implementação e avaliação de enfermagem. Foram utilizados os livros NANDA e NIC como sistemas de classificação de diagnósticos e intervenções, respectivamente. Aprovado pelo Parecer CAEE: 42321514.4.0000.0021 CEP/UFMS de 31 de março de 2015. Resultados: puérpera em terceiro dia de pós-operatório, Gesta 3 Parto Cesáreo 3 Aborto 1. Apresentou as patologias DM1 e Hipertensão gestacional. A partir da descrição da anamnese e exame físico, foram elencados os diagnósticos: Risco de glicemia instável relacionada à gestação; Nutrição desequilibrada: menor do que as necessidades corporais relacionada à ingestão alimentar insuficiente caracterizado por picos de hipoglicemia; Risco de quedas relacionado às alterações fisiológicas na glicemia sanguínea; e Volume de líquidos excessivo caracterizado por alteração na pressão arterial, edema, desequilíbrio eletrolítico e ansiedade. Após, houve o planejamento e implementação das ações de cuidado, com enfoque no monitoramento e controle da glicose e volume sanguíneo. Conclusão: a organização da assistência com a SAE, propicia o enfoque nas especificidades e demandas iminentes da paciente, possibilitando a integralidade do cuidado. 